\title{
Fungicidal activity and PK/PD of caspofungin as tools to guide antifungal therapy in a fluconazole-resistant $C$. parapsilosis candidemia
}

Carlo Tascini ${ }^{\mathrm{a}}$, Emanuela Sozio ${ }^{\mathrm{b}}$, Antonello Di Paolo ${ }^{\mathrm{c}}$, Giancarlo Tintori ${ }^{\mathrm{b}}$, Alessandro Leonildi ${ }^{\mathrm{a}}$, Giacomo Bertolino ${ }^{\mathrm{d}}$, Franco Carmassi ${ }^{\mathrm{b}}$, Enrico Tagliaferri ${ }^{\mathrm{a}}$, Francesco Menichetti ${ }^{\mathrm{a}}$, Francesco Barchiesi $^{\mathrm{e}}$

${ }^{a}$ Infectious Diseases Unit, Azienda Ospedaliera Universitaria Pisana, Via Paradisa 2, 56124 Pisa, Italy

${ }^{\mathrm{b}}$ Emergency Medicine Unit, Azienda Ospedaliera Universitaria Pisana, Via Paradisa 2, 56124 Pisa, Italy

${ }^{\mathrm{c}}$ Department of Clinical and Experimental Medicine, Via Roma 55, 56126 Pisa, Italy

${ }^{\mathrm{d} D e p a r t m e n t ~ o f ~ P h a r m a c e u t i c a l ~ S c i e n c e s-M e d i c i n e ~ M a n a g e m e n t, ~ A z i e n d a ~ O s p e d a l i e r a ~}$

Universitaria Pisana, Via Paradisa 2, 56124 Pisa, Italy

${ }^{\mathrm{e} I n f e c t i o u s ~ D i s e a s e s ~ C l i n i c, ~ U n i v e r s i t a ̀ ~ P o l i t e c n i c a ~ d e l l e ~ M a r c h e, ~ V i a ~ C o n c a ~ 71, ~} 60126$ Torrette (AN), Italy 


\begin{abstract}
C. parapsilosis may be responsible for bloodstream infections (BSI) and it is characterized by an increased incidence of fluconazole resistance. A 75-year old woman with severe comorbidities received the insertion of a peripherally-inserted central venous catheter. Fluconazole did not prevent a $C$. parapsilosis BSI hence caspofungin was started after a nephrotoxic first-line treatment with amphotericin B. The ratio of peak plasma concentration over the minimum inhibitory concentration $\left(\mathrm{C}_{\mathrm{max}} / \mathrm{MIC}\right)$ ratio was adopted to maximize efficacy of caspofungin. MIC and plasma Cmax values were obtained by broth microdilution and LC-MS, respectively. Interestingly, daily doses of 1 $\mathrm{mg} / \mathrm{kg}$ (total daily dose, $50 \mathrm{mg}$ ) allowed the achievement of $\mathrm{C}_{\max } / \mathrm{MIC}$ values $>10$. The optimized regimen was safe and effective, leading to negative blood culture at day 8 . The patient was discharge at home at day 21 . Therefore, individualized dosing regimens of caspofungin may be effective and safe even in the case of $C$. parapsilosis BSI.
\end{abstract}

Keywords: C. parapsilosis; fluconazole resistance; caspofungin; PK/PD; dose personalization; therapeutic drug monitoring 


\section{Introduction}

C. parapsilosis is the second or third most common Candida species isolated from bloodstream infections (BSI) worldwide [1,2]. Although fluconazole is generally effective in infections caused by this Candida spp., an increasing incidence of fluconazole resistance has been lately documented among clinical isolates of $C$. parapsilosis [3]. Caspofungin has potent antifungal activity and it has been shown to be as effective as, and less toxic than amphotericin B in the treatment of invasive candidiasis $[4,5]$. Interestingly, minimum inhibitory concentration (MIC) values of the echinocandin for C. parapsilosis are higher than those for other Candida spp. due to polymorphisms of genes encoding for beta-glucan synthase (i.e., FKS1 and FKS2) [6, 7]. The reduced susceptibility of $C$. parapsilosis for echinocandin was not associated with an increased incidence of treatment failure in clinical practice [8], even if some studies reported a relationship between the clinical failure of echinocandins and higher MIC values [9]. Additional dosefractionation studies showed that the in vivo fungicidal activity of echinocandins is maximized against $C$. albicans isolates when the ratio of maximum plasma drug concentration to MIC $\left(\mathrm{C}_{\max } / \mathrm{MIC}\right)$ approaches 10 [10]. Nevertheless, there are insufficient data to support a correlation between echinocandin susceptibility testing and clinical outcome, while antibiogram results may widely differ among laboratories.

Overall, these data (i.e., high prevalence of C. parapsilosis, its natural reduced susceptibility to echinocandins, and higher rate of fluconazole resistance) would make infections due to this species difficult to manage.

In the present paper, we describe the case report of a woman with a blood stream infection due to $C$. parapsiolosis and its effective treatment with caspofungin. Of note, drug dosage was optimized by the application of a therapeutic drug monitoring protocol. 


\section{Case presentation}

The case report concerns a 75-year-old female patient, whose medical history was significant for systemic sclerosis with diffuse vasculopathy, pulmonary hypertension, gastrointestinal disorders (i.e.: recurrent diarrhoea), joints and macro-vascular involvement, mitral and tricuspid valvulopathy, chronic renal failure, and hypogammaglobulinaemia. She was diagnosed a candida oesophagitis and treated with oral fluconazole for almost 3 months (daily dose of $100 \mathrm{mg}$; total dose of $9 \mathrm{~g})$. The occurrence of dysphagia required the parenteral nutrition through a peripherallyinserted central venous catheter (PICC). Other pharmacological therapies consisted of daily methylprednisolone and immunoglobulins on a monthly basis. Three months later, the patient was admitted to emergency department because of onset of fever, dysphagia and worsening dyspnoea. Laboratory analyses revealed leukocytosis (12320 cells $/ \mathrm{mm}^{3}$, neutrophils $84 \%$ ) and increased procalcitonin $(27.81 \mathrm{ng} / \mathrm{ml})$, while chest X-ray showed the presence of right basal pneumonia. The patient was treated with non-invasive mechanical ventilation with continuous positive airway pressure and subsequently admitted to an Internal Medical ward. Sputum, urine and blood samples were taken for microbiological analyses, therapy with piperacillin/tazobactam was started, while fluconazole $200 \mathrm{mg} /$ day was continued.

Blood cultures were performed by the BACTEC 860 system (Becton-Dickinson, Inc., Sparks, MD), while the specie was identified using API ID 32C system (bioMerieux, Marcy l'Etoile, France). MIC values were determined by Sensititre YeastOne (Thermoscientific), according to Clinical \& Laboratory Standards Institute (CLSI) clinical breakpoints cut-off values for susceptibility. Furthermore, actual values of MICs and minimal fungicidal concentration (MFC) for caspofungin were determined by a broth microdilution method in accordance with CLSI document M27-A3 [11]. Briefly, plates were incubated at $35^{\circ} \mathrm{C}$ for $24 \mathrm{~h}$ and readings were performed visually. Caspofungin MICs were considered as the lowest concentrations of the antifungal agent at which well turbidity was $50 \%$ of controls or less. After MIC readings, the plates were centrifuged at 2000 rpm, the supernatant was discarded, and each well was added with $50 \mu \mathrm{L}$ of sterile saline solution. 
The saline solution of those wells whose caspofungin concentration was higher than the MIC value was transferred in duplicate onto a $150-\mathrm{mm}$ Sabouraud dextrose agar plate and incubated at $35^{\circ} \mathrm{C}$ for $24 \mathrm{~h}$. MFC value was defined as the lowest drug concentration associated with a killing $>99 \%$ and each test was run in quadruplicate. Antifungal susceptibility testing was performed as described above, with and without 50\% human serum (obtained from healthy volunteers) in the medium.

Cultures of blood samples taken the day after the admission confirmed a BSI caused by $C$.

parapsilosis (first from PICC than from peripheral vein). Liposomal amphotericin B (3 mg/Kg once daily) did substitute fluconazole, and the PICC was removed from the vein without evidence of septic thrombophlebitis. Subsequent in vitro susceptibility results yielded the following MIC values: flucytosine $0.06 \mathrm{mg} / \mathrm{L}$, amphotericin B $0.25 \mathrm{mg} / \mathrm{L}$, anidulafungin $1 \mathrm{mg} / \mathrm{L}$, caspofungin 0.5 $\mathrm{mg} / \mathrm{L}$, micafungin $0.5 \mathrm{mg} / \mathrm{L}$, itraconazole $0.5 \mathrm{mg} / \mathrm{L}$, voriconazole $2 \mathrm{mg} / \mathrm{L}$, and fluconazole $32 \mathrm{mg} / \mathrm{L}$. Both fundus examination and echocardiography were negative, while white blood cell count, Creactive protein, and procalcitonin normalized despite the presence of fever. However, a progressive worsening of renal function (creatinine increase, from $1.15 \mathrm{~g} / \mathrm{dL}$ to $2.8 \mathrm{~g} / \mathrm{dL}$ ) occurred the fifth day after the hospitalization, so that caspofungin represented the drug of choice. In order to achieve a maximum plasma concentration $\left(\mathrm{C}_{\mathrm{max}}\right) / \mathrm{MIC}$ value $\geq 10$ [10], caspofungin loading and maintenance doses were $70 \mathrm{mg}$ and $50 \mathrm{mg} /$ day (as $1 \mathrm{mg} / \mathrm{kg} /$ day), respectively. Therapeutic drug monitoring started on the third day; caspofungin plasma concentrations were measured $1 \mathrm{~h}$ after the dose $\left(\mathrm{C}_{\max }\right)$ and $1 \mathrm{~h}$ immediately before the next administration (minimum plasma concentration, $\mathrm{C}_{\min }$ ). The measurement of caspofungin plasma concentrations was performed by a liquid chromatographymass spectrometry instrument (Waters TQD, Waters, Milford, MA, USA) using a commercially available kit (Chromsystems $\mathrm{GmbH}$, Munich, Germany). The plasma $\mathrm{C}_{\max }$ and $\mathrm{C}_{\min }$ values accounted for 13.9 and $3.0 \mathrm{mg} / \mathrm{L}$, respectively, and Table 1 reports caspofungin susceptibility results obtained by broth microdilution method in quadruplicate. Geometric mean values of MIC and MCF for the isolate $\# 11236$ were 0.06 and $1.0 \mathrm{mg} / \mathrm{L}$, respectively, showing that $\mathrm{C}_{\max } / \mathrm{MIC}$ ratio 
was 231.6. Interestingly, caspofungin susceptibility significantly decreased for both isolates in the presence of $50 \%$ of serum in the test medium (4-fold and 2-fold increase in MIC and MCF values, respectively; Table 1). Moreover, the $\mathrm{C}_{\max } / \mathrm{MIC}$ ratio decreased up to 12.7 in the presence of serum, but that value was greater than the pharmacokinetic/pharmacodynamic (PK/PD) threshold [10], suggesting that the administration of the chosen maintenance dose could result in an effective treatment. In the same in vitro conditions, the $\mathrm{C}_{\max } / \mathrm{MFC}$ ratio decreased from 13.9 up to 6.0 (Table $1)$.

The blood culture yielded a negative result on day $8^{\text {th }}$ of caspofungin administration. The patient fully recovered after two additional weeks of therapy, and she finally left the hospital. Although our data are based only on a case of BSI due to a C. parapsilosis highly susceptible to caspofungin in vitro, we confirmed that a good $\mathrm{PK} / \mathrm{PD}$ ratio was related to a positive outcome of the infection. It's interesting to note that even when the fungicidal parameter was considered and the $\mathrm{PK} / \mathrm{PD}$ ratio decreased (i.e., $\mathrm{C}_{\max } / \mathrm{MFC}$ equal to 13.9 or 6.0 without or with serum, respectively), the magnitude was still near the optimal achievable effect (i.e.: greater than 10). Finally, caspofungin susceptibility testing performed by the commercial system yielded MIC higher than those obtained by the reference broth microdilution method $(0.5 v s 0.06 \mathrm{mg} / \mathrm{L})$ while it was similar to the MFC obtained by the same method $(1.0 \mathrm{mg} / \mathrm{L})$.

\section{Discussion}

Fluconazole resistance among C.parapsilosis isolates is not yet a rare event and appears to be increasing wordwide [3]. Pfaller et al. showed that isolates of C. parapsilosis isolates from nosocomial and community onset BSI ( $5.8 \%$ and $6.6 \%$, respectively) were resistant to fluconazole [12]. Therefore, guidelines suggest to consider antifungals other than fluconazole as therapy for invasive candidiasis in patients who have had prior azole exposure and who are unstable or infected with species known to have reduced susceptibilities to fluconazole [13]. 
After a long-lasting exposure to fluconazole, the present patient developed a fluconazole-resistant isolate that initially responded to amphotericin B. However, the macrolide administration caused the early onset of a renal toxicity. Caspofungin was an effective alternative to amphotericin B, because the daily dose was chosen according to target values of $\mathrm{PK} / \mathrm{PD}$ parameters. Caspofungin displays a concentration-dependent activity [14], and both free area-under-the-time-concentration curve $(f \mathrm{AUC}) / \mathrm{MIC}$ and $\mathrm{C}_{\max } / \mathrm{MIC}$ ratios are predictive parameters of drug efficacy [14-16]. In particular, $\mathrm{C}_{\max } / \mathrm{MIC}$ values higher than 4 could predict an improved clinical outcome [17]. Therefore, the evaluation of strain sensitivity coupled with therapeutic drug monitoring allows the development of dosing strategies that could optimize treatment outcomes for antimicrobial drugs, as the present case has demonstrated.

\section{Conclusions}

In conclusion, caspofungin was an effective option to amfotericin B halted for its nephrotoxic effects, while the once-daily administration of echinocandins ensures the highest plasma peak concentrations. However, the application of $\mathrm{PK} / \mathrm{PD}$ parameters for the optimization of antifungal therapies is still poorly utilised despite their usefulness. Finally, the fungicidal activity of caspofungin in the presence or not of serum might be useful information for the clinicians who treat life-threatening candidemia, especially in frail patients. 


\section{Competing interest}

The Authors declare that they have no competing interests. 


\section{References}

1. Pfaller MA, Diekema DJ, Gibbs DL, Newell VA, Ng KP, Colombo A, et al. Geographic and temporal trends in isolation and antifungal susceptibility of Candida parapsilosis: a global assessment from the ARTEMIS DISK Antifungal Surveillance Program, 2001 to 2005. J Clin Microbiol 2008;46(3):842-9.

2. Bassetti M, Taramasso L, Nicco E, Molinari MP, Mussap M, Viscoli C. Epidemiology, species distribution, antifungal susceptibility and outcome of nosocomial candidemia in a tertiary care hospital in Italy. PLoS One 2011;6(9):e24198.

3. Tortorano AM, Prigitano A, Dho G, Grancini A, Passera M, Group E-FS. Antifungal susceptibility profiles of Candida isolates from a prospective survey of invasive fungal infections in Italian intensive care units. J Med Microbiol 2012;61(3):389-93.

4. Mora-Duarte J, Betts R, Rotstein C, Colombo AL, Thompson-Moya L, Smietana J, et al. Comparison of caspofungin and amphotericin B for invasive candidiasis. N Engl J Med 2002;347(25):2020-9.

5. Spellberg BJ, Filler SG, Edwards JE, Jr. Current treatment strategies for disseminated candidiasis. Clin Infect Dis 2006;42(2):244-51.

6. Ostrosky-Zeichner L, Rex JH, Pappas PG, Hamill RJ, Larsen RA, Horowitz HW, et al. Antifungal susceptibility survey of 2,000 bloodstream Candida isolates in the United States. Antimicrob Agents Chemother 2003;47(10):3149-54 .

7. Fleck R, Dietz A, Hof H. In vitro susceptibility of Candida species to five antifungal agents in a German university hospital assessed by the reference broth microdilution method and Etest. J Antimicrob Chemother 2007;59(4):767-71.

8. Fernandez-Ruiz M, Aguado JM, Almirante B, Lora-Pablos D, Padilla B, Puig-Asensio M, et al. Initial use of echinocandins does not negatively influence outcome in Candida 
parapsilosis bloodstream infection: a propensity score analysis. Clin Infect Dis 2014;58(10):1413-21.

9. Cheung C, Guo Y, Gialanella P, Feldmesser M. Development of candidemia on caspofungin therapy: a case report. Infection 2006;34(6):345-8.

10. Andes D, Marchillo K, Lowther J, Bryskier A, Stamstad T, Conklin R. In vivo pharmacodynamics of HMR 3270, a glucan synthase inhibitor, in a murine candidiasis model. Antimicrob Agents Chemother 2003;47(4):1187-92.

11. CLSI. Reference Method for Broth Dilution Antifungal Susceptibility testing of Yeast; Approved Standard-Third Edition. CLSI document M27-A3. Wayne, PA: Clinical and Laboratory Standard Institute; 2008. 2008.

12. Pfaller MA, Diekema DJ, Andes D, Arendrup MC, Brown SD, Lockhart SR, et al. Clinical breakpoints for the echinocandins and Candida revisited: integration of molecular, clinical, and microbiological data to arrive at species-specific interpretive criteria. Drug Resist Updat 2011;14(3):164-76

13. Pappas PG, Rex JH, Sobel JD, Filler SG, Dismukes WE, Walsh TJ, et al. Guidelines for treatment of candidiasis. Clin Infect Dis 2004;38(2):161-89.

14. Wiederhold NP, Kontoyiannis DP, Chi J, Prince RA, Tam VH, Lewis RE. Pharmacodynamics of caspofungin in a murine model of invasive pulmonary aspergillosis: evidence of concentration-dependent activity. J Infect Dis 2004;190(8):1464-71.

15. Louie A, Deziel M, Liu W, Drusano MF, Gumbo T, Drusano GL. Pharmacodynamics of caspofungin in a murine model of systemic candidiasis: importance of persistence of caspofungin in tissues to understanding drug activity. Antimicrob Agents Chemother 2005;49(12):5058-68.

16. Lepak A, Castanheira M, Diekema D, Pfaller M, Andes D. Optimizing Echinocandin dosing and susceptibility breakpoint determination via in vivo pharmacodynamic evaluation against 
Candida glabrata with and without fks mutations. Antimicrob Agents Chemother. 2012;56(11):5875-82.

17. Mazzei T, Novelli A. Pharmacological properties of antifungal drugs with a focus on anidulafungin. Drugs 2009;69(Suppl 1):79-90. 
Table 1. Caspofungin susceptibilities of the C. parapsilosis isolate \#11236 tested by a broth microdilution method.

\begin{tabular}{|l|l|l|}
\hline Testing & Culture medium & Culture medium plus serum \\
\hline MIC $^{*}(\mathrm{mg} / \mathrm{L})$ & $0.06(0.06-0.06)$ & $1.1(1.0-2.0)$ \\
\hline $\mathrm{MFC}^{*}(\mathrm{mg} / \mathrm{L})$ & $1.0(1.0-1.0)$ & $2.3(2.0-4.0)$ \\
\hline $\mathrm{C}_{\max } / \mathrm{MIC}$ ratio & 231.6 & 12.7 \\
\hline $\mathrm{C}_{\max } / \mathrm{MFC}$ ratio & 13.9 & 6.04 \\
\hline
\end{tabular}

Notes: ${ }^{*}$, results are expressed as geometric mean (range).

Abbreviations: MIC, minimum inhibitory concentration; MFC, minimum fungicidal concentration; $\mathrm{C}_{\max }$, maximum plasma concentration. 